DOI: $10.17516 / 1999-494 X-0275$

УДК 517.977 .56

\title{
Optimization of
}

\section{Distributed Information-Measuring Systems}

\author{
Nikolay D. Demidenko ${ }^{a, b}$ and Ludmila V. Kulagina*b \\ ${ }^{a}$ Federal Research Center for Information \\ and Computational Technologies Krasnoyarsk Branch \\ Krasnoyarsk, Russian Federation \\ ${ }^{b}$ Siberian Federal University \\ Krasnoyarsk, Russian Federation
}

Received 29.10.2020, received in revised form 03.11.2020, accepted 30.11.2020

Abstract. The article presents the task of optimizing the information-measuring system for controlling objects with distributed parameters. The optimal control problem is formulated using the functions of distributed control (measurement). The necessary optimality conditions are obtained. Based on these conditions, a numerical algorithm for calculating the optimal functions of distributed control is constructed. Numerical experiments were carried out.

Keywords: mathematical modeling, optimal control systems, distributed control functions.

Citation: Demidenko N.D., Kulagina L.V. Optimization of distributed information-measuring systems, J. Sib. Fed. Univ. Eng. \& Technol., 2020, 13(8), 923-932. DOI: 10.17516/1999-494X-0275

(C) Siberian Federal University. All rights reserved

This work is licensed under a Creative Commons Attribution-Non Commercial 4.0 International License (CC BY-NC 4.0).

* Corresponding author E-mail address: klvation@gmail.com 


\title{
Оптимизация распределенных
}

\section{информационно-измерительных систем}

\author{
Н.Д. Демиденко ${ }^{\mathbf{a}, \boldsymbol{0}}$, Л.В. Кулагина ${ }^{\tilde{0}}$ \\ ${ }^{a}$ Федеральный исследовательский центр \\ информациионых и вычислительных технологий \\ Красноярский филиал \\ Российская Федерачия, Красноярск \\ ${ }^{6}$ Сибирский федеральный университет \\ Российская Федераиия, Красноярск
}

\begin{abstract}
Аннотация. Рассмотрена задача оптимизации информационно-измерительной системы для управления объектами с распределенными параметрами. Сформулирована задача оптимального управления с использованием функций распределенного контроля (измерения). Получены необходимые условия оптимальности. На основе этих условий построен численный алгоритм вычисления оптимальных функций распределенного контроля. Проведены численные эксперименты.

Ключевые слова: математическое моделирование, системы оптимального управления, функции распределенного контроля.
\end{abstract}

Цитирование: Демиденко, Н.Д. Оптимизация распределенных информационно-измерительных систем / Н.Д. Демиденко, Л.В. Кулагина // Журн. Сиб. федер. ун-та. Техника и технологии, 2020. 13(8). С. $923-932$. DOI: $10.17516 / 1999-494 X-0275$

Поскольку объект - основное звено системы регулирования, правильная организация сбора информации о состоянии сложного распределенного процесса является ключом к рациональному решению проблемы управления. Распределенный характер процесса требует также применения распределенного контроля. Это подтверждается успешной практикой использования контроля в средней точке.

Для оптимального управления распределенными процессами более важен контроль в некоторых средних точках объекта, чем на выходе из аппарата. На практике при управлении отдельными распределенными процессами или сложными технологическими системами, составляющими цепочки аппаратов, ведется контроль разных параметров процесса в различных точках системы, полученная информация должна обрабатываться с целью формирования соответствующего управляющего сигнала.

Информационной системой распределенного контроля мы называем систему, состоящую из множества датчиков, расположенных в различных точках пространственно-распределенного объекта, соединенных с ними устройств измерения и первичной обработки информации, и предназначенную для оценки состояния объекта.

\section{Введение}

Возможно, более правильным было бы говорить об информационной системе распределенного измерения. Однако мы будем употреблять ранее принятую терминологию [1], следуя традиции, закрепленной к настоящему времени большим числом работ. 
Система распределенного контроля, содержащая $n$ линейных точечных датчиков, характеризуется вектором распределенного контроля: $\gamma=\left(x_{1}, \ldots, x_{n} ; g_{1}, \ldots, g_{n}\right)$, где $x_{i}$ - координаты установки датчиков; $g_{1}, \ldots, g_{n}$ - коэффициенты усиления датчиков. Теоретически, если датчики устанавливаются во всех точках области $D$, мы приходим к понятию системы непрерывно-распределенного контроля, которая характеризуется весовой функцией распределенного контроля $g(x) \in \Omega$.

Аналогичным будет представление о системе распределенного воздействия как о совокупности устройств, установленных в различных точках управляемого объекта с распределенными параметрами и предназначенных для изменения состояния объекта.

Система распределенного воздействия вместе с управляющим устройством (которое получает информацию от системы распределенного контроля) образует систему распределенного управления. При увеличении объема поступающей информации необходимо освободить человека-оператора как слабое звено в системе. Это обусловлено не только возрастанием непосильной нагрузки на человека, но и субъективностью суждений оператора при оценке состояния процесса. Необходима строго обоснованная автоматическая оценка поступающей информации о состоянии процесса, а также рациональная организация самой системы контроля.

Достаточно общая задача состоит в том, что требуется найти оптимальную оценку состояния данного процесса для формирования регулирующих воздействий по каждому каналу по данным распределенного контроля. Найденная оптимальная оценка состояния процесса дает решение рациональной организации системы контроля для получения необходимой информации. В частности, может быть полезна идея оптимизации систем управления типовыми технологическими процессами, состоящая в следующем.

Предполагается, что используемый класс управляющих устройств ограничен и большей частью состоит из типовых регуляторов. Решается задача установления наиболее рационального способа контроля управляемого объекта, обеспечивающего при данных условиях наилучшее управление им. Другими словами, оптимизируется процесс управления, прежде всего за счет удачного выбора критерия состояния процесса и использования распределенного контроля.

В наиболее простом случае эта задача приводит к нахождению той или иной весовой функции распределенного контроля. Тогда реализация систем управления сводится к установке на объекте некоторого количества датчиков, сигналы которых определенным образом усредняются и поступают на управляющее устройство. Условная схема системы изображена на рис. 1.

В более сложном случае при управлении учитывается изменение не только самих функций, характеризующих процесс в объекте, но и их производных (скоростей) по временно́й и пространственной координате.

Результаты исследований [1-3] показали, что применение данного метода дает возможность повысить качество управления процессов при использовании сравнительно простых технических средств. Таким образом, с помощью рациональной организации распределенного контроля могут решаться важные задачи оптимизации систем регулирования.

\section{Постановка задачи}

Мы рассмотрим простейшие математические постановки задач оптимального сбора информации в замкнутых автоматических системах, содержащих объекты с распределенными

$$
-925-
$$




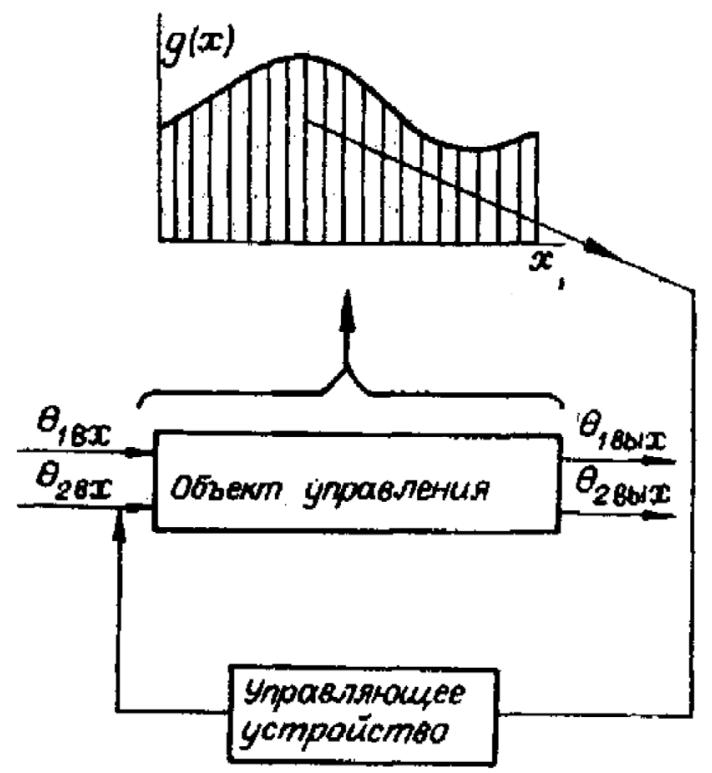

Рис. 1. Принципиальная схема использования непрерывно-распределенного контроля

Fig. 1. Schematic diagram of continuously distributed control application

параметрами. В системах управления технологическими объектами большинство используемых датчиков по своим характеристикам близко к точечным. Тем не менее полезно начать обсуждение вопросов оптимального сбора информации в системах с распределенными параметрами системы, содержащей распределенный датчик.

Способ обработки информации в такой системе простейший, он заключается в интегрировании с некоторым весом функции состояния объекта по всей области пространства, в которой распределен процесс.

Такая постановка оправдана, во-первых, тем, что в некоторых производствах все же используют датчики с распределенными параметрами (например, при производстве стеклопластиков применяют датчик, распределенный по длине объекта [4]). Во-вторых, она позволяет выяснить предельные возможности улучшения качества управляемых систем за счет рационального сбора информации, поскольку оценка состояния объекта, сформулированная указанным способом, является наиболее общей в классе линейных безынерционных оценок. В-третьих, имея весовую функцию распределенного контроля, можно поставить задачу об «аппроксимации» этой функции некоторой решетчатой функцией, что соответствует замене распределенного датчика конечным числом точечных датчиков. Подобный путь хотя в принципе и возможен, однако не кратчайший.

Постановка задач об отыскании оптимальных весовых функций распределенного контроля представляет и методический интерес, поскольку обнаруживается общность задач оптимального сбора информации с задачами оптимального управления пространственно-распределенными объектами. Поэтому для решения задач оптимального контроля можно использовать математический аппарат теории оптимального управления объектами с распределенными параметрами. 
Управляемый процесс описывается следующей системой дифференциальных уравнений:

$$
\begin{aligned}
& \frac{\partial \theta_{1}(x, t)}{\partial t}+\omega_{1} \frac{\partial \theta_{1}(x, t)}{\partial x}=k_{1}\left(\theta_{1}(x, t)-\theta_{2}(x, t)\right)+f(x) w(t) \\
& \frac{\partial \theta_{2}(x, t)}{\partial t}-\omega_{2} \frac{\partial \theta_{2}(x, t)}{\partial x}=k_{2}\left(\theta_{2}(x, t)-\theta_{1}(x, t)\right)
\end{aligned}
$$

где $f(x) w(t)$ - внешнее воздействие с управляемой функцией $w(t) ; w_{i},(i=1,2)$ - скорость течения взаимодействующих сред; $k_{i},(i=1,2)$ - теплофизические параметры; $\alpha_{\mathrm{i}}=\frac{k_{i}}{w_{i}}-$ обобщенные параметры объекта.

Дополним систему (1) начальными

$$
\begin{aligned}
& \left.\theta_{1}(x, t)\right|_{t=0}=\theta_{1}(x), \\
& \left.\theta_{2}(x, t)\right|_{t=0}=\theta_{2}(x)
\end{aligned}
$$

и граничными условиями

$$
\begin{aligned}
& \theta_{1}(0, t)=\theta_{1 \mathrm{~B} 1}(t), \\
& \theta_{2}(1, t)=\theta_{2 \mathrm{~B} 2}(t) .
\end{aligned}
$$

Система (1)-(3) представляет собой простейшую математическую модель управляемого процесса с распределенными параметрами, например динамику теплообменных аппаратов с тонкими стенками.

Будем полагать целью системы управления объектов (1)-(3) минимизацию функционала качества

$$
F=\int_{0}^{\mathrm{T}}\left(\theta^{*}-\theta_{1 \text { вых }}(t)\right) \mathrm{d} t
$$

где $\theta^{*}$ - заданное значение управляемого параметра.

Переходный процесс в регулируемой среде в оригиналах можно представить следующей формулой:

$$
\begin{aligned}
& \theta_{1}(x, t)=\int_{0}^{\mathrm{t}} \mathrm{G}_{11}(x, \mathrm{t}, \tau) \theta_{1 \mathrm{BX}}(\tau) \mathrm{d} \tau+ \\
& +\int_{0}^{t}\left(G_{12}(x, \dot{t}, \tau) v(\tau)+\breve{G}_{12}(x, t, \tau) w(\tau)\right) \mathrm{d} \tau
\end{aligned}
$$

где $G_{11}, G_{12}, \breve{G}_{12}$ - импульсные переходные функции, представляющие собой реакцию объекта на воздействие в виде $\delta$-функции по соответствующим каналам воздействий. Оба регулятора определяются функциями

$$
v(\tau)=-\int_{0}^{t} u_{1}(t, \tau) \varphi_{1}(\tau) \mathrm{d} \tau, \quad w(t)=-\int_{0}^{t} u_{2}(t, \tau) \varphi_{2}(\tau) \mathrm{d} \tau,
$$

где $u_{1}(t, \tau), u_{2}(t, \tau)$ - ядра, которые считаются заданными; $\varphi_{1}(t)$ и $\varphi_{2}(t)$ - сигналы, поступающие на регуляторы и выражающиеся следующими соотношениями:

$$
\varphi_{\mathrm{i}}(\tau)=\int_{0}^{1} \theta_{1}(x, \tau) g_{i}(x) \mathrm{d} x, i=1,2
$$

где $g_{i}(x)$ - функция распределенного контроля (измерения). 
Ставится задача нахождения таких функций распределенного контроля $g_{1}(x)$ и $g_{2}(x)$, которые минимизируют функционал качества (4).

Подставим (6) в $\theta_{1}(x, t)$ и получим

$$
\theta_{1}(x, t)=\int_{0}^{t} G_{11}(x, t, \tau) \theta_{1 \mathrm{BX}}(\tau) d \tau-\int_{0}^{1} \int_{0}^{t}\left(g(\xi), M\left(x, t, \xi, \tau, \theta_{1}\right)\right) \mathrm{d} \tau \mathrm{d} \xi,
$$

где введены следующие векторные функции:

$$
\begin{aligned}
& g(\xi)=\left(g_{1}(\xi), g_{2}(\xi)\right), \\
& M\left(x, t, \xi, \tau, \theta_{1}\right)=\left(M_{1}\left(x, t, \xi, \tau, \theta_{1}\right), M_{2}\left(x, t, \xi, \tau, \theta_{1}\right)\right), \\
& M_{1}\left(x, t, \xi, \tau, \theta_{1}\right)=\theta_{1}(\xi, \tau) \int_{0}^{t} G_{12}(x, t, \eta) u_{1}(\eta, \tau) \mathrm{d} \eta, \\
& M_{2}\left(x, t, \xi, \tau, \theta_{1}\right)=\theta_{1}(\xi, \tau) \int_{0}^{t} \breve{G}_{12}(x, t, \eta) u_{2}(\eta, \tau) \mathrm{d} \eta .
\end{aligned}
$$

Поварьируем оптимальную весовую вектор-функцию $g(x)$ :

$$
\tilde{g}(x)= \begin{cases}g(x), & x \in[0,1] / \delta \\ G, & x \in \delta\end{cases}
$$

где $g(x)$ и $G \in \Omega ; \delta$ - сколь угодно малый отрезок внутри $[0,1]$, длина которого равна $\varepsilon$.

Функционал качества $F$ получит следующее значение:

$$
\begin{aligned}
& F(\tilde{g})=\int_{0}^{\mathrm{T}}\left(\theta^{*}-\left(\theta_{\text {1вых }}(t)+\Delta \theta_{1 \text { вых }}(t)\right)\right)^{2} \mathrm{~d} t= \\
& =\int_{0}^{\mathrm{T}}\left(\left(\theta^{*}-\theta_{1 \text { вых }}(t)\right)^{2}-2\left(\theta^{*}-\theta_{1 \text { вых }}(t)\right) \Delta \theta_{1 \text { вых }}(t)\right) \mathrm{d} t .
\end{aligned}
$$

После несложных тождественных преобразований найдем

$$
\begin{aligned}
& \Delta \theta_{1 \text { вых }}(t)=-\varepsilon\left(\left(G-g\left(\xi^{\prime}\right)\right),\left(\int_{0}^{T} M\left(1, t, \xi, \tau, \theta_{1}\right) d \tau+\right.\right. \\
& \left.+\int_{0}^{1} \int_{0}^{t} \Gamma(1, t, \xi, \tau) \int_{0}^{\tau} M\left(\xi, \tau, \eta, \theta_{1}\right) \mathrm{d} \eta \mathrm{d} \tau \mathrm{d} \xi\right),
\end{aligned}
$$

где резольвента $\Gamma(x, t, \xi, \tau)$ удовлетворяет уравнению

$$
\begin{aligned}
& \Gamma(x, t, \xi, \tau)=\left(g(\xi), \frac{\partial M\left(x, t, \xi, \tau, \theta_{1}\right)}{\partial \theta_{1}}\right)- \\
& -\int_{0}^{1} \int_{0}^{t} \Gamma(\zeta, \eta, \xi, \tau)\left(g(\zeta), \frac{\partial M\left(x, t, \zeta, \eta, \theta_{1}\right)}{\partial \theta_{1}}\right) \mathrm{d} \eta \mathrm{d} \zeta .
\end{aligned}
$$

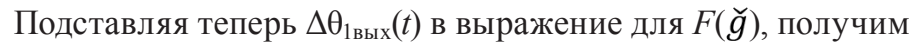

$$
F(\tilde{g})=F(g)-2 \varepsilon\left(\Pi\left(\xi^{\prime}, G\right)-\Pi\left(\xi^{\prime}, g\left(\xi^{\prime}\right)\right),\right.
$$

где 


$$
\begin{aligned}
& \Pi\left(\xi^{\prime}, G\right)==-\left(G,\left(\int _ { 0 } ^ { \mathrm { T } } ( \theta ^ { * } - \theta _ { 1 \text { вых } } ( \mathrm { t } ) ) \left(\int_{0}^{\mathrm{t}} M\left(1, \mathrm{t}, \xi^{\prime}, \tau, \theta_{1}\right) \mathrm{d} \tau-\right.\right.\right. \\
& \left.\left.\left.-\int_{0}^{1} \int_{0}^{\mathrm{t}} \Gamma(1, \mathrm{t}, \xi, \tau) \int_{0}^{\tau} M\left(\xi, \tau, \xi^{\prime}, \eta, \theta_{1}\right) \mathrm{d} \eta \mathrm{d} \tau \mathrm{d} \xi\right) \mathrm{dt}\right)\right), \\
& \Pi\left(\xi^{\prime}, g\left(\xi^{\prime}\right)\right)=-\left(g\left(\xi^{\prime}\right),\left(\int _ { 0 } ^ { T } ( \theta ^ { * } - \theta _ { 1 \text { вых } } ( t ) ) \left(\int_{0}^{t} M\left(1, t, \xi^{\prime}, \tau, \theta_{1}\right) \mathrm{d} \tau-\right.\right.\right. \\
& \left.\left.\left.-\int_{0}^{1} \int_{0}^{t} \Gamma(1, t, \xi, \tau) \int_{0}^{\tau} M\left(\xi, \tau, \xi^{\prime}, \eta, \theta_{1}\right) \mathrm{d} \eta \mathrm{d} \tau \mathrm{d} \xi\right) \mathrm{d} t\right)\right) .
\end{aligned}
$$

Далее имеем

$$
\Delta F=F(\tilde{g})-F(g)=-2 \varepsilon\left(\Pi\left(\xi^{\prime}, G\right)-\Pi\left(\xi^{\prime}, g\left(\xi^{\prime}\right)\right)\right) \geq 0,
$$

откуда $\Pi\left(\xi^{\prime}, G\right) \leq \Pi\left(\xi^{\prime}, g\left(\xi^{\prime}\right)\right), g\left(\xi^{\prime}\right) \in \Omega, \xi^{\prime} \epsilon[0,1]$.

Поскольку $g(x)$ - оптимальная вектор-функция, а $G$ - произвольный элемент $\Omega$, то последнее условие означает, что для оптимальности $g(x)$ необходимо, чтобы функция П $(\xi, g(\xi))$ достигала максимума при любом фиксированном $\xi$.

Если $\Omega$ определяют неравенства $\left|g_{i}(x)\right| \leq 1, i=1,2$, получаем

$$
\begin{aligned}
& g_{i}(x)=\operatorname{sign}\left\{-\int_{0}^{T}\left(\theta^{*}-\theta_{1 \text { вых }}(t)\right)\left(\int_{0}^{t} M_{i}\left(1, \mathrm{t}, x, \tau, \theta_{1}\right) \mathrm{d} \tau-\right.\right. \\
& \left.\left.-\int_{0}^{1} \int_{0}^{t} \Gamma(1, t, \xi, \tau) \int_{0}^{\tau} M_{i}\left(\xi, \tau, x, \eta, \theta_{1}\right) \mathrm{d} \eta \mathrm{d} \tau \mathrm{d} \xi\right) \mathrm{d} t\right\} \quad i=1,2 .
\end{aligned}
$$

\section{Результаты численного моделирования, их обсуждение и выводы}

Рассмотрим результаты численного анализа переходных процессов и весовых функций распределенного измерения в объектах с распределенными параметрами в замкнутой системе регулирования при распределенном управляющем воздействии. Эти результаты сравнивают с кривыми переходных процессов и весовыми функциями распределенного измерения без распределенного управления, когда управление осуществляли только за счет изменения граничных условий (при регулирующем воздействии, поступающем только на вход объекта).

Весовые функции $g_{1}(x)$ и $g_{2}(x)$ находили из полученных необходимых условий оптимальности методом последовательных приближений.

На рис. 2 и 3 представлены кривые переходных процессов и весовых функций, получающихся на каждой итерации. Анализ переходных процессов проводили для параметров технологических аппаратов $\alpha_{1} \in[0,10]$ и $\alpha_{2} \in[0,10]$. Время регулирования $T=2,0$. Как показали расчеты, для устойчивых переходных процессов итеративный процесс довольно быстро сходится. В пределах требуемой точности обычно делают 5-6 итераций. Кривые, обозначенные 

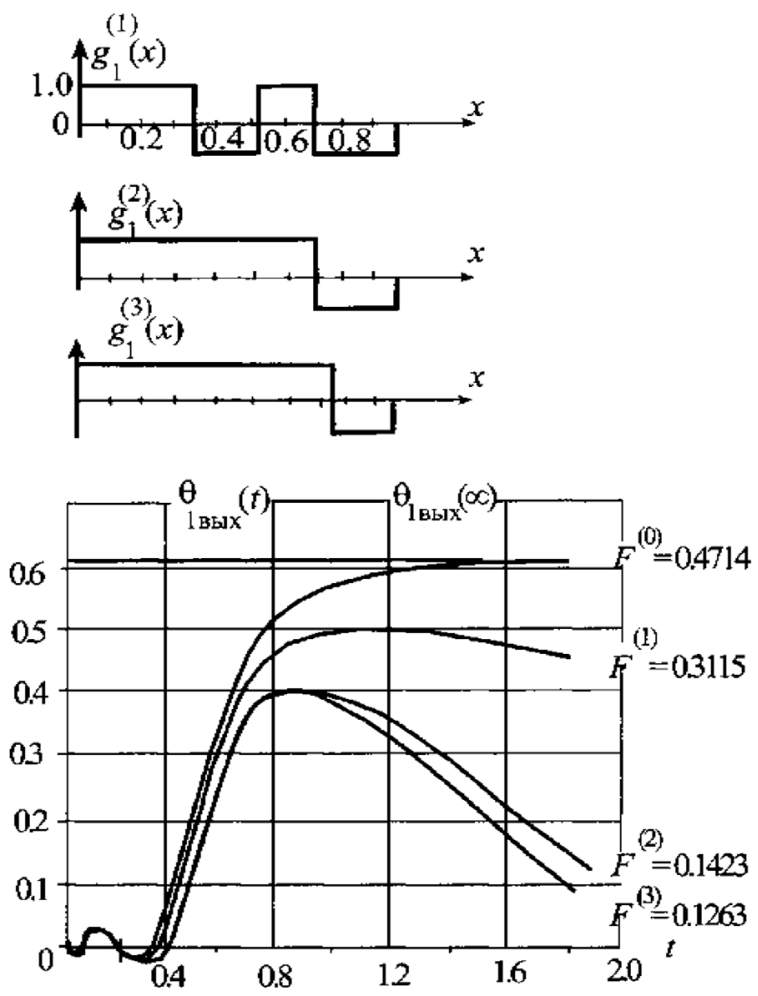

Рис. 2. Кривые регулирования в одноконтурной системе [5]

Fig. 2. Control curves in a single-loop system [5]

$F^{(0)}$, соответствуют переходному процессу в разомкнутой системе регулирования. При этом новое установившееся значение выходной величины совпадает с полученными стационарными значениями по методу инерционности.

Проведенные расчеты показывают, что качество регулирования при распределенном управляющем воздействии повышается. Так, для параметров $\alpha_{1}=1, \alpha_{2}=2$ (см. рис. 2) показатель качества $F^{\text {опт }}=0,1263$ без распределенного воздействия, а при распределенном управлении (рис. 3) с подачей управляющего воздействия в точку аппарата с координатой $x_{\mathrm{i}}=0,7$ $F^{\text {опт }}=0,0949$. При этом показатель качества улучшился примерно на $20 \%$.

Ниже приведем описание блок-схемы программы расчета оптимальных весовых функций, соответствующих функций переходного процесса и функционала качества системы регулирования. Основные этапы вычислительного алгоритма:

1) задается начальное приближение весовых функций распределенного контроля $g_{i}^{0}(l)$, $i=1,2, \ldots, n$;

2) вычисляются значения функций $\theta_{1}(0, t), \theta_{1}(1, t), \Delta \theta_{1}(0, t), \Delta \theta_{1}(1, t)$ в замкнутой системе регулирования;

3) по формуле (4) рассчитывается значение функционала $F$;

4) по формулам (7) определяются новые значения функций $g_{i}(l)$;

$5)$ уточняются функции $g_{i}(l), i=1,2, \ldots, n$, начиная с пункта 2 , до совпадения с заданной точностью двух последовательных приближений. 

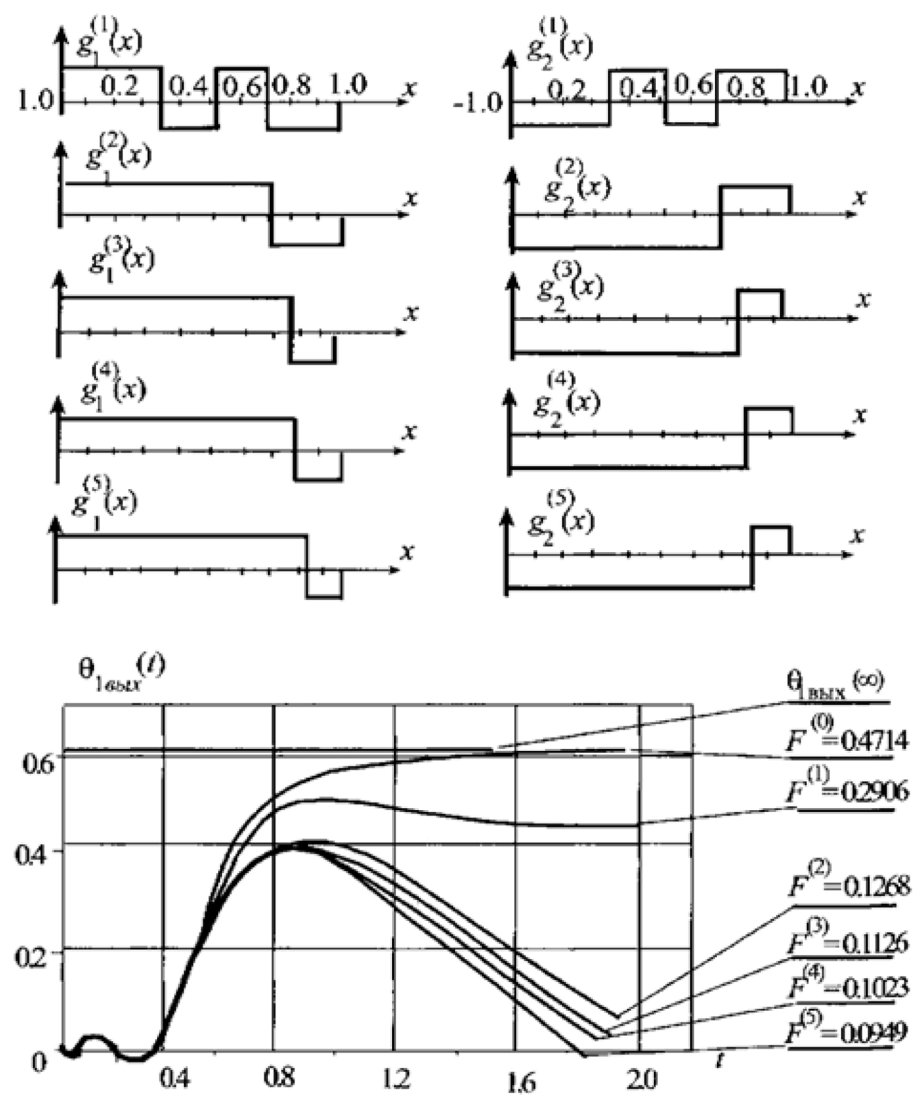

Рис. 3. Кривые регулирования в двухконтурной системе [5]

Fig. 3. Control curves in dual-system [5]

Исследования показывают, что алгоритм достаточно быстро сходится к искомым решениям.

\section{Благодарности / Acknowledgments}

Исследование выполнено при финансовой поддержке РФФИ и Правительства Красноярского края в рамках научных проектов № 18-48-242001 «Теплофизические и гидродинамические особенности кинетики смесеобразования при иммобилизации радиоактивных отходов в цементную матрицу с использованием эффектов кавитации» и 18-41-242004 «Теоретические основы кондиционирования вод питьевого назначения на базе эффектов гидродинамической кавитации».

The reported study was funded by RFBR and the government of Krasnoyarsk region according to the research projects no. 18-48-242001 Thermo physical and hydrodynamic features of the kinetics of mixture formation upon immobilization of radioactive waste in cement matrix using the effects of cavitation \& 18-41-242004 Theoretical Foundations of potable water conditioning on the basis of the effects of hydrodynamic cavitation. 


\section{Список литературы / References}

[1] Демиденко Н.Д. Моделирование и оптимизация тепломассообменных процессов в химической технологии. М.: Наука, 1991. 240 c. [Demidenko N.D. Modeling and optimization of heat and mass transfer processes in chemical technology. Moscow: Nauka, 1991. 240 p. (in Russian)]

[2] Демиденко Н.Д. Управляемые распределенные системы. Новосибирск: Наука, 1999. 393 c. [Demidenko N.D. Managed Distributed Systems. Novosibirsk: Nauka, 1999. 393 p. (in Russian)]

[3] Демиденко Н.Д., Кулагин В.А., Шокин Ю.И. Моделирование и вычислительные технологии распределенных систем. Новосибирск: Наука, 2012. 424 с. [Demidenko N.D., Kulagin V.A., Shokin Y.I. Modeling and computing technology of distributed systems. Novosibirsk: Nauka, 2012. 424 p. (in Russian)]

[4] Бугров А.В., Дудкин Н.И., Масленников И.М. Об управлении химико-технологическими объектами с пространственно распределенными параметрами с использованием емкостных измерительных преобразователей. Автоматизация химических производств. М.: НИИТЭХИМ, 1973. Вып. 1. С. 34-47. [Bugrov A.V., Dudkin N.I., Maslennikov I.M. About management of chemical-technological objects with spatially distributed parameters using capacitive measuring transducers. In book. Automation of chemical production. Moscow: NIITEHIM, 1973. Vol. 1. P. 34-47 (in Russian)]

[5] Demidenko N.D., Kulagina L.V., Pyanykh A.A. Optimal control of systems with distributed parameters, J. Sib. Fed. Univ. Eng. technol. 2019, 12(8), 903-915. DOI: 10.17516/1999-494X-0190 (in Russian). 